\title{
Synthesis, Characterization and Biological Applications of Water-Soluble ZnO Quantum Dots
}

\author{
Raphaël Schneider ${ }^{1}$, Lavinia Balan ${ }^{2}$ and Fadi Aldeek ${ }^{1}$ \\ ${ }^{1}$ Laboratoire Réactions et Génie des Procédés (LRGP), \\ Nancy-University, CNRS, Nancy, \\ 2Institut de Science des Matériaux de Mulhouse (IS2M), Mulhouse,
}

France

\section{Introduction}

Quantum dots (QDs) or semiconductor nanocrystals are of great interest to fundamental studies but have also potential applications as biological probes (Medintz et al., 2005), fluorescent biosensor (Costa-Fernandez et al., 2006), light-emitting diodes (LEDs) (Lim et al. 2007), and solar cells (Robel et al., 2006). Owing to the effect of quantum confinement, QDs show exceptional physical and chemical properties such as sharp and symmetrical emission spectra, high quantum yield (QY), good photo- and chemical stability, and size-dependent emission-wavelength tunability (Bruchez et al., 1998; Chan et al., 1998). For biological labelling, the most studied QDs are the nanocrystals of CdSe and CdTe (Aldeek et al., 2008) and the corresponding core/shell structured QDs (such as CdSe/ZnS, CdTe/ZnS or $\mathrm{CdTe} / \mathrm{ZnTe}$ ) that are more robust against chemical degradation or photooxidation than the parent cores (Law et al., 2009). Recent findings have highlighted the acute toxicity of II-VI semiconductor QDs without an external layer of a nontoxic material on biological systems (Schneider et al., 2009; Dumas et al. 2010). This toxicity results mainly from the decomposition and release of heavy metal ions and formation of reactive oxygen species. The toxicity of cadmium is a concern that will also limit the use of these visible or near IR emitting nanocrystals, especially for applications directly related to human health. Synthesis of low toxicity QDs and especially Cd-free QDs is the most challenging aspect of working with these materials in biological and medical fields. A promising member of the Cd-free QD is $\mathrm{ZnO}$. However, $\mathrm{ZnO}$ nanoparticles are not stable in water. This instability is related to their surface luminescent mechanisms. Water molecules are able to attack the luminescent centers on the $\mathrm{ZnO}$ surface and destroy them rapidly. This chapter describes the strategies that have been developed over the last years to transfer ZnO QDs in water.

\section{ZnO photoluminescence}

For the past two decades, study of $\mathrm{ZnO}$ nanoparticles has become a leading edge in nanotechnology. With the reduction of size, novel electrical, chemical and optical properties are introduced, which are largely believed to be the result of surface and quantum confinement effects (Ahmad et al., 2011). 


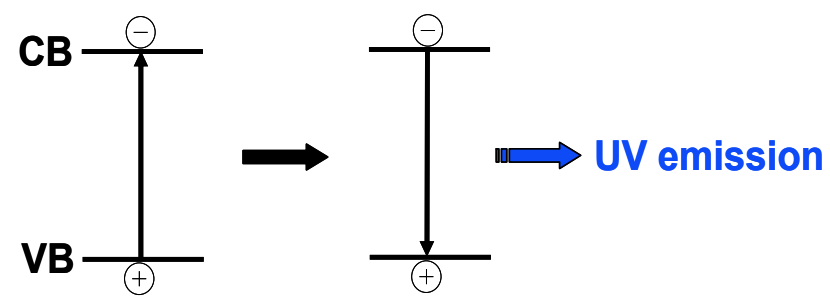

Fig. 1. Exciton emission of $\mathrm{ZnO}$ quantum dots.

At room temperature, wurtzite-ZnO QDs exhibit dual emission bands in the near UV and visible range (Djurisic et al., 2006; Zhang et al., 2010; Xiong, 2010). The UV emission at about $370 \mathrm{~nm}$ (band gap $=3.37 \mathrm{eV}$ at room temperature) is associated with the radiative recombination of an electron from the conduction band with a hole from the valence band (band-edge emission) (Figure 1) or in a trap near the valence band (near-band-edge emission). Because the recombination of the photo-generated electron with a hole in the valence band is directly associated with the band gap, it is a size-dependent emission due to quantum confinement.

The origin of the visible luminescence (also called deep-level emission) is more complicated, its mechanism is controversial and not fully understood. Numerous hypotheses have been proposed to explain the visible luminescence such as oxygen vacancies $\left(\mathrm{V}_{\mathrm{O}}\right)$, zinc vacancies $\left(\mathrm{V}_{\mathrm{Zn}}\right)$, oxygen intersticials $\left(\mathrm{O}_{\mathrm{i}}\right)$, zinc intersticials $\left(\mathrm{Zn}_{\mathrm{i}}\right)$, and antisite oxygen $\left(\mathrm{O}_{\mathrm{Zn}}\right)$ (Zhang et al., 2010). Among them, two mechanisms involving oxygen vacancies are the most cited. The first one is the recombination of a shallowly trapped electron with a hole in a deep trap (Figure 2a). The second one is the recombination of an electron in a singly occupied oxygen vacancy with a photo-generated hole in the valence band (Figure 2b) (Xiong, 2010). Additionnally, copper and alkaline metals impurities, surface-bonded hydroxides, and donor-acceptor complexes have also been proposed to be responsible of the visible photoluminescence.

(a)

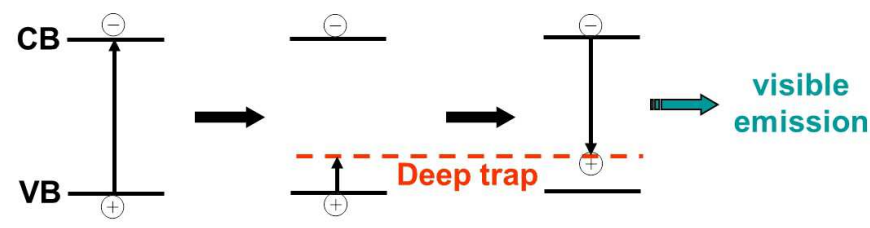

(b)

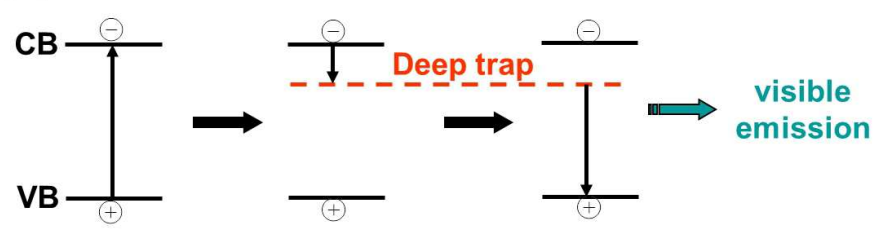

Fig. 2. Defect emission of $\mathrm{ZnO}$ quantum dots. 


\section{Synthesis and biological applications of $\mathrm{ZnO}$ quantum dots}

Because the final materials are highly cristallised and of high purity, physical methods such as chemical vapour deposition (An et al., 2006), pulsed laser deposition (Choi et al., 2001), and chemical vapour transport and condensation processes (Ding et al., 2004) are the most popular methods to produce $\mathrm{ZnO}$ QDs with fine UV emission (Djurisic et al., 2006). However, these routes are not free of disadvantages and harsh conditions are often required preventing these methods from being used for large-scale production and their wide application.

Moreover, visible emission is the most-suited for biomedical and bio-imaging applications because most cells and tissues appear blue under UV light.

$\mathrm{ZnO}$ QDs with high defect concentrations are therefore the most appropriate for such applications. Because the sol-gel technique syntheses have many advantages such as process simplicity and high homogeneity, and that they offer the possibility of preparing large quantities of small-sized ZnO QDs at low cost and at low temperature, they constitute one of the best choice to obtain $\mathrm{ZnO}$ QDs with highly visible emission. Decomposition of organozinc derivatives and electrolysis constitute new and alternative methods for the production of green-yellow emitting $\mathrm{ZnO}$ QDs and will be described at the end of this paragraph.

\subsection{The sol-gel synthesis of $\mathrm{ZnO}$ quantum dots}

The sol-gel process can briefly be defined as the conversion of a precursor solution into an inorganic solid by chemical means. The sol-gel route for the production of ZnO QDs involves generally the basic hydrolysis of zinc acetate $\mathrm{Zn}(\mathrm{OAc})_{2}$ mediated by an alkaline hydroxide or tetramethylammonium hydroxide (TMAH) in an alcohol (Figure 3) (Bahnemann et al., 1987; Spanhel et al., 1991).

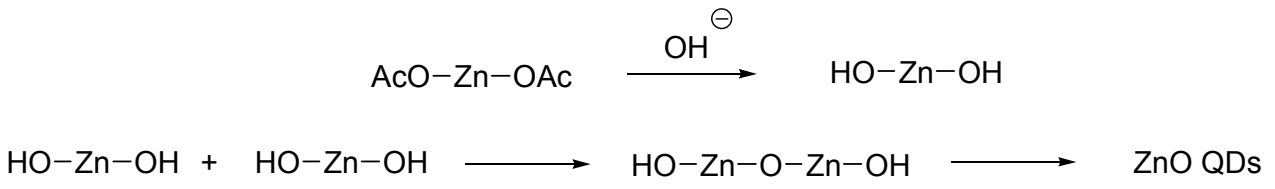

Fig. 3. The sol-gel synthesis of $\mathrm{ZnO}$ nanoparticles.

After the nucleation stage, the $\mathrm{ZnO}$ photoluminescence (PL) exhibits a continuous red-shift and its quantum yield (QY) decreases gradually (van Dijken et al., 2001). Protective organic ligands must therefore be added on the $\mathrm{ZnO}$ surface to inhibit further crystal growth and aggregation. Polymers, alkoxysilanes, oleic acid and triethanolamine are the most commonly stabilisers used to coordinate with $\mathrm{Zn}$ atoms on the surface of $\mathrm{ZnO}$ QDs to hinder the formation of bulk $\mathrm{ZnO}$.

\subsubsection{Polymer-capped ZnO QDs}

The conventional sol-gel technique of $\mathrm{ZnO}$ synthesis was first modified in 2005 to prepare $\mathrm{ZnO}$ nanocrystals stabilized by poly(ethylene glycol)methyl ether (PEGME). Zinc salts $\left[\mathrm{CH}_{3}\left(\mathrm{CH}_{2} \mathrm{CH}_{2} \mathrm{O}\right) \mathrm{nCH}_{2} \mathrm{COO}\right]_{2} \mathrm{Zn}$ were hydrolysed with $\mathrm{LiOH}$ in ethanol to get $\mathrm{ZnO}(\mathrm{PEGME})$ QDs with diameters ranging from 1 to $4 \mathrm{~nm}$ and PL tunable from blue to yellow depending on the $[\mathrm{LiOH}] /[\mathrm{Zn}]$ ratio used for the synthesis (Xiong et al., 2005; Xiong et al., 2005). 
Although stabilized by hydrophilic poly(ethyleneglycol) moieties, the water-dispersability of these ZnO QDs has not been studied.

Poly(ethyleneimine) (PEI) can also be used to disperse ZnO QDs in water with preservation of their optical properties (Joshi et al., 2009). ZnO QDs, produced by hydrolysis of Zn(OAc) with $\mathrm{LiOH}$, were first stabilized by trisodium citrate through hydrogen bonding and then treated by PEI to get stable dispersions of the dots in water (Figure 4).

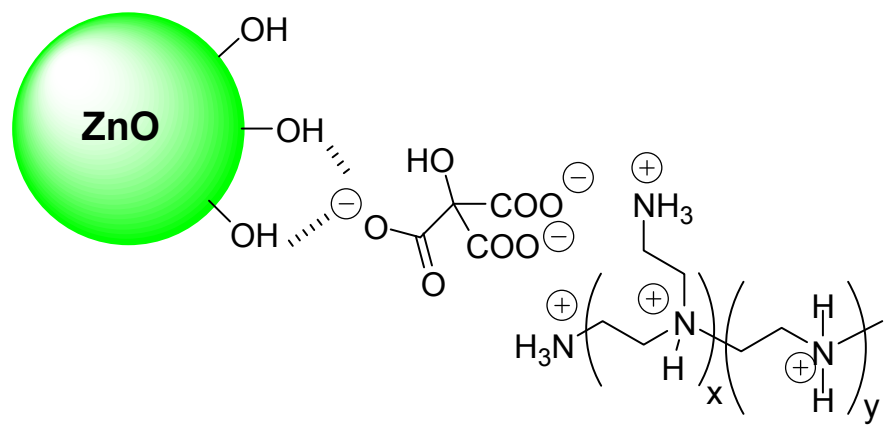

Fig. 4. Proposed mechanism for the stabilization of $\mathrm{ZnO} Q D$ s using sodium citrate and PEI.

ZnO QDs produced by this method are ca. $5 \mathrm{~nm}$ in diameter, exhibit a strong green-yellow fluorescence at $555 \mathrm{~nm}$ upon excitation at $360 \mathrm{~nm}$ and were found to be stable in aqueous solution for at least 45 days.

Coating ZnO QDs with a polymer shell is an efficient method for stabilizing the photophysical properties of the cores. Xiong et al. were the first to report the preparation of core/shell $\mathrm{ZnO} /$ polymer QDs by initiating the polymerization with the inherent free radicals pre-existing on ZnO QDs surfaces (Xiong et al., 2006). For that purpose, zinc methacrylate was hydrolysed with $\mathrm{LiOH}$ to obtain blue-emitting methacrylate-coated $\mathrm{ZnO}$ QDs. Copolymerisation of these nanoparticles with methyl methacrylate for instance affords uniform and monodisperse $\mathrm{ZnO}$ core QDs with an inorganic core diameter of $2.1 \mathrm{~nm}$. The thickness of the polymer shell is about $1 \mathrm{~nm}$. Once dispersed in organic solvents, the PL QY of ZnO@polymer QDs reached 80\%.

A similar strategy was employed by the authors to prepare water-stable ZnO QDs (Xiong et al., 2007). Poly(methacrylic acid)(PMMA)-capped ZnO QDs were co-polymerised with methacrylic acid to produce ZnO@PMMA microspheres of about $150 \mathrm{~nm}$ in diameter that exhibit blue fluorescence (PL QY $=22 \%$ ) once dispersed in water. From the viewpoint of practical applications, such large nanoparticles are not suitable for biological and medical fields, especially for labelling functional subcellular or proteins, as larger particles with diameters above $25 \mathrm{~nm}$ often affect their biological function and are more likely to be recognized and cleared by the phagocyte (Medintz et al., 2005).

The first successful labelling of living cells with ZnO QDs was only described in 2008 (Xiong et al., 2008). Green- or yellow-emitting ZnO QDs were prepared by basic hydrolysis of zinc methacrylate using $\mathrm{LiOH}$ or $\mathrm{NaOH}$, respectively, and concomitant polymerisation with poly(ethylene glycol)methyl ether methacrylate. Final ZnO QDs have a copolymer shell with the internal hydrophobic polymethacrylate layer protecting the nanocrystals and the external hydrophilic poly(ethylene glycol)methyl ether groups allowing their transfer in aqueous medium (Figure 5). 


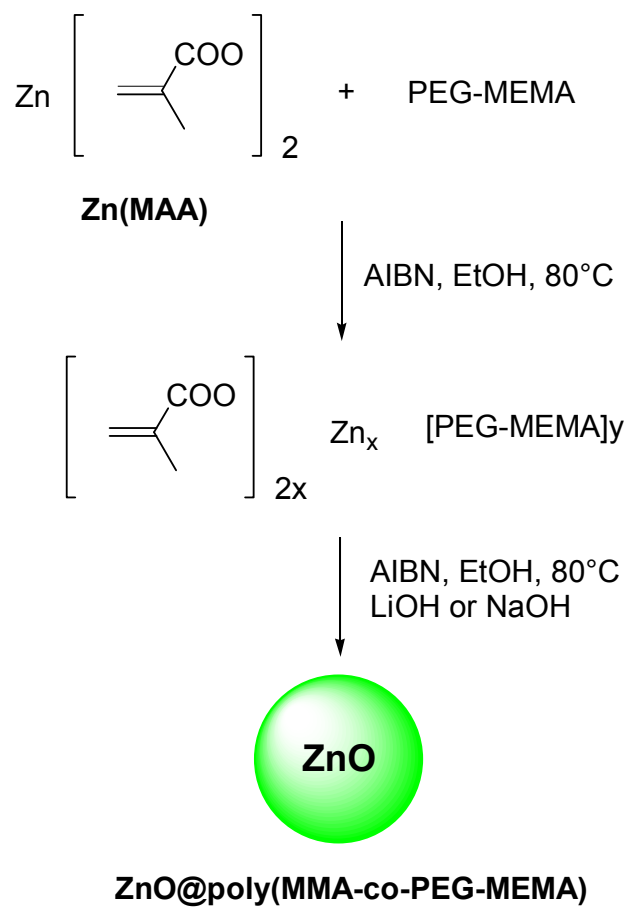

Fig. 5. Synthesis of ZnO@poly(MMA-co-PEG-MEMA) quantum dots.

The obtained nanocrystals have diameters of ca. 3-4 $\mathrm{nm}$ and exhibit a stable photoluminescence in water (up to $50 \%$ PL QY for QDs prepared with LiOH). Efficient labelling of the cytoplasm of human hepatoma cells was achieved using aqueous dispersions of these dots at a concentration of $0.1 \mathrm{mg} / \mathrm{mL}$.

Small-sized polymer-coated ZnO QDs were also recently prepared by hydrolysis of zinc methacrylate by $\mathrm{LiOH}$ and concomitant AIBN-initiated polymerisation with 2(dimethylaminoethyl) ethylmethacrylate (DMAEMA) (Figure 6) (Zhang et al., 2010).

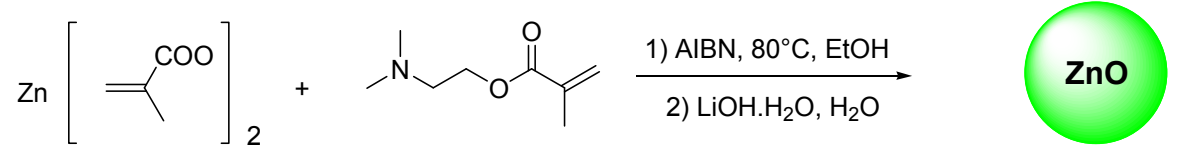

Fig. 6. Synthesis of ZnO@poly(MMA-DMAEMA) quantum dots.

The obtained QDs are ca. $4 \mathrm{~nm}$ in diameter, exhibit a strong yellow luminescence and have a PL QY of 21\%. Positively-charged ZnO@poly(MMA-DMAEMA) QDs were found to associate with negatively charged plasmid DNA ( $\mathrm{p}$-DNA) to form spherical particles around $200 \mathrm{~nm}$ in diameter. Using the Luciferase reporter gene, it was found that QD/p-DNA complexes could efficiently transfect COS-7 cells and allowed real-time imaging of gene transfection in live cells (Figure 7). 


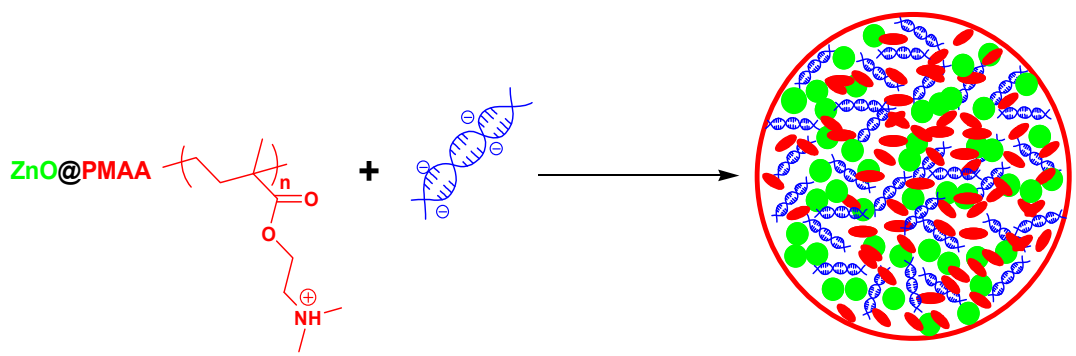

Fig. 7. Schematic of QD/pDNA complex formation.

$\mathrm{ZnO}$ QDs have also successfully been integrated into polymer (chitosan - $\mathrm{M}_{\mathrm{w}} \approx 200 \mathrm{kDa}-$ and acrylic acid) nanospheres (Yan et al., 2011). In that one-pot process, chitosan, $\mathrm{Zn}\left(\mathrm{NO}_{3}\right)_{2}$ and acrylic acid were first heated at $80^{\circ} \mathrm{C}$ and $\mathrm{ZnO}$ QDs formed by hydroxide ions generation from chitosan. Polymerisation was initiated by addition of potassium persulfate $\mathrm{K}_{2} \mathrm{~S}_{2} \mathrm{O}_{8}$ and chitosan finally cross-linked using glutaraldehyde. The obtained chitosan-ZnO QDs nanospheres have an average diameter of ca. $150 \mathrm{~nm}$ and $\mathrm{ZnO}$ QDs were found to be homogeneously distributed in the shell of the nanospheres. A strong blue-emission centered at $440 \mathrm{~nm}$ could only be observed for these nanospheres using high amounts of $\mathrm{Zn}\left(\mathrm{NO}_{3}\right)_{2}$ relative to chitosan.
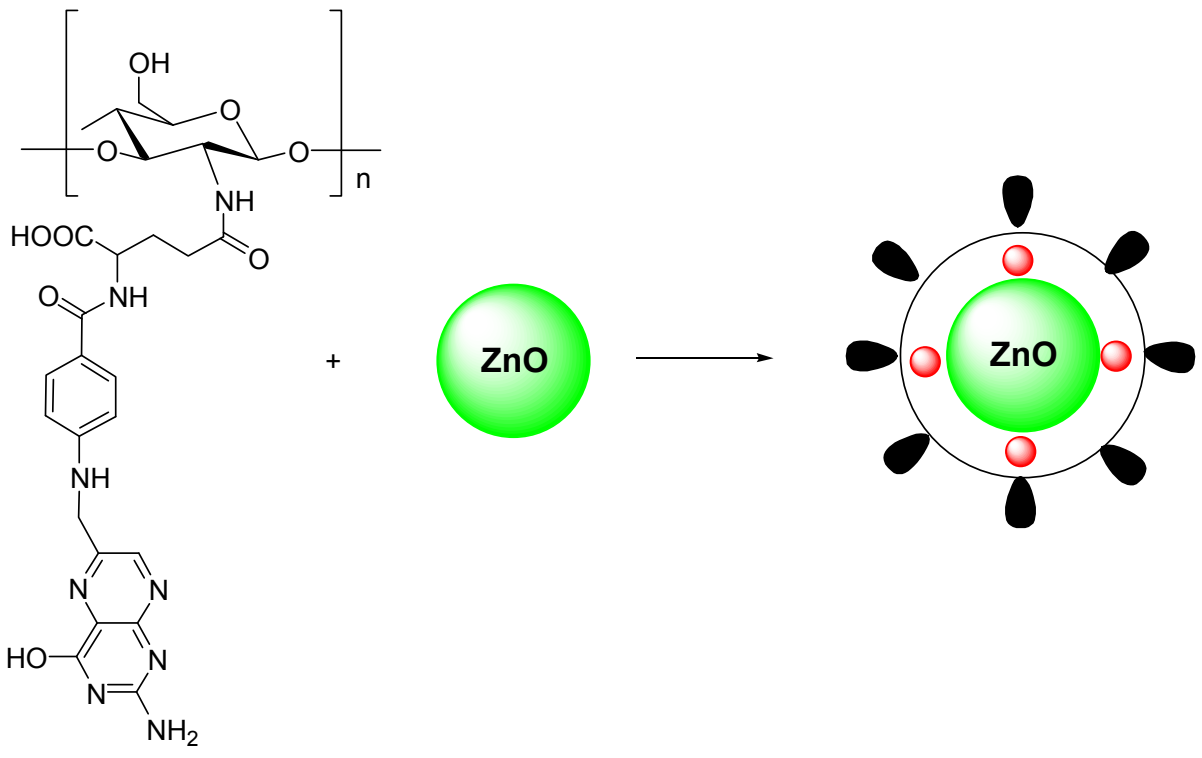

Folate-linked chitosan

$O=$ folic acid $\quad O=$ Doxorubicin

Fig. 8. Preparation of $\mathrm{ZnO}$ quantum dots encapsulated by folate-conjugated chitosan. 
The natural and biodegradable polymer chitosan was also used to encapsulate and stabilize $\mathrm{ZnO}$ QDs through electrostatic interactions. Chitosan $\left(\mathrm{M}_{\mathrm{w}} \approx 50 \mathrm{kDa}\right)$ was first conjugated to folic acid and then associated to $\mathrm{ZnO}$ QDs produced by hydrolysis of $\mathrm{Zn}(\mathrm{OAc})_{2}$ with an aqueous solution of diethanolamine in the presence of oleic acid used as stabilizer (Yuan et al., 2010). The obtained ZnO QDs have an average diameter of $3 \mathrm{~nm}$, exhibit strong blue fluorescence at $440 \mathrm{~nm}$, and were found to be stable in water for at least one year. The "ZnO-chitosan-folate" platform can associate Doxorubicin, an antineoplastic agent used in tumor treatments, through physical and chemical interactions and thus be used as nanocarrier (Figure 8). The drug-release responses at $\mathrm{pH}=5.3$ or 7.4 were characterized by an initial rapid drug release (ca. $85 \%$ within $36 \mathrm{~h}$ ) followed by a controlled release.

\subsubsection{Silanization of ZnO QDs}

An alternative and interesting methodology for surface-functionalization and stabilization of $\mathrm{ZnO}$ nanocrystals is based on organosilanes chemistry to inhibit decomposition in aqueous media. Since the first report of $\mathrm{ZnO}$ nanoparticles surface modification with silane, this strategy has successfully been used for the stabilisation of $\mathrm{ZnO}$ QDs in water. Trialkoxysilanes have a strong affinity with hydroxylated surfaces, form covalent bonds with them, thereby creating a shielding barrier of cross-linked polysiloxanes that protects the nanocrystal at the core (Bruce et al., 2005; Soares et al., 2008).

$\mathrm{ZnO}$ nanoparticles with diameters of ca. $20 \mathrm{~nm}$ (hydrodynamic diameter of $60 \mathrm{~nm}$ ) can simply be prepared by heating of $\mathrm{Zn}(\mathrm{OAc})_{2} \cdot 2 \mathrm{H}_{2} \mathrm{O}$ in methanol at $68^{\circ} \mathrm{C}$ for $7 \mathrm{~h}$. These QDs were immediately capped by 3-aminopropyltrimethoxysilane (APTES) to inhibit the particle growth at the end of the synthesis. After excitation at $350 \mathrm{~nm}$, ZnO@APTES nanocrystals show two emission bands at ca. 380 and $560 \mathrm{~nm}$. A second capping $\left(\mathrm{SiO}_{2}\right.$ or $\left.\mathrm{TiO}_{2}\right)$ was introduced through the sol-gel process at the periphery of ZnO@APTES nanocrystals to improve the PL intensity and to red-shift the fluorescence emission peaks. Optimal $\mathrm{SiO}_{2}$ or $\mathrm{TiO}_{2}$ shell thickness in both cases was found to be $0.5 \mathrm{~nm}$ (Wu et al., 2007). Once a $\mathrm{SiO}_{2}$ shell introduced at the periphery of ZnO@APTES QDs, the visible green-yellow emission was found to be markedly increased. When ZnO@APTES QDs were capped with $\mathrm{TiO}_{2}$, the PL emission was slightly red-shifted to $520-590 \mathrm{~nm}$. A high PL QY was also obtained for core/shell $\mathrm{ZnO} / \mathrm{TiO}_{2}$ nanocrystals $(79 \%$ in $\mathrm{MeOH})$. When mung beens seeds were germinated in the presence of these $\mathrm{ZnO}$ colloidal dispersions, a strong fluorescence in the cell walls of the vascular cylinder was observed, indicating a good uptake of the nanocrystals and their good potential in bio-imaging studies.

Another report of silazination to get small-sized, water-dispersible and stable ZnO QDs was made in 2007 (Jana et al., 2007). Oleate-stabilized ZnO QDs produced by hydrolysis of $\mathrm{Zn}(\mathrm{OAc})_{2}$ with tetramethylammonium hydroxide (TMAH) were treated by $\mathrm{N}-(2-$ aminoethyl)aminopropyltrimethoxysilane (AEAPS) and TMAH in a two-stage silanization process to prepare dots with an average diameter of ca. $5 \mathrm{~nm}$ (PL QY $=16-20 \%$ in water) (Figure 9). This two-step silanization was developed to minimize polysiloxane by-products formation during the surface-capping of ZnO QDs.

QDs with a mixed shell containing amine and phosphonate groups at their periphery were also prepared by this method to vary the surface charge and thus the isoelectric points of the nanocrystals. The primary amine groups of the AEAPS ligand were also found to contribute to the photostability of $\mathrm{ZnO}$ QDs, probably through interaction of the nitrogen atoms with surface zinc atoms. This property was used for the detection of various aldehydes, including 
the biologically important glucose and acetaldehyde, by photoluminescence. Imine functions formed upon reaction of peripheral $\mathrm{NH}_{2}$ functions with aldehydes induced a marked photostability decrease (up to $71 \%$ with $o$-phthaldehyde).
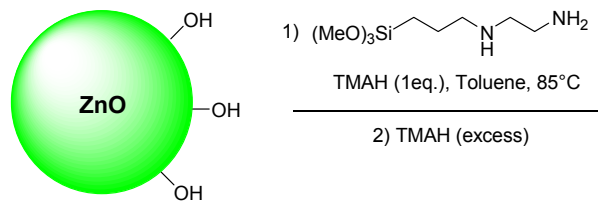

TMAH (1eq.), Toluene, $85^{\circ} \mathrm{C}$

2) TMAH (excess)

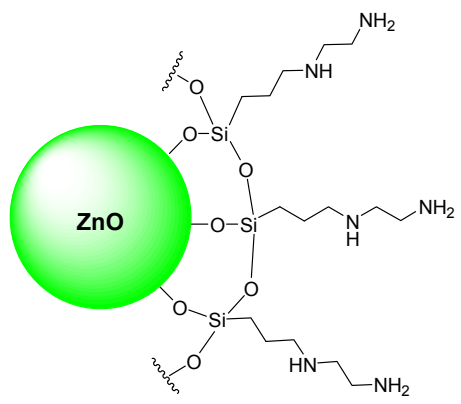

Fig. 9. Synthesis of ZnO@AEAPS quantum dots.

Our group used as similar strategy to anchor 2-[ethoxy(polyethyleneglycol)propyl] triethoxysilane at the surface of hydrophobic oleate-capped $\mathrm{ZnO}$ QDs (Figure 10) (Moussodia et al., 2008).
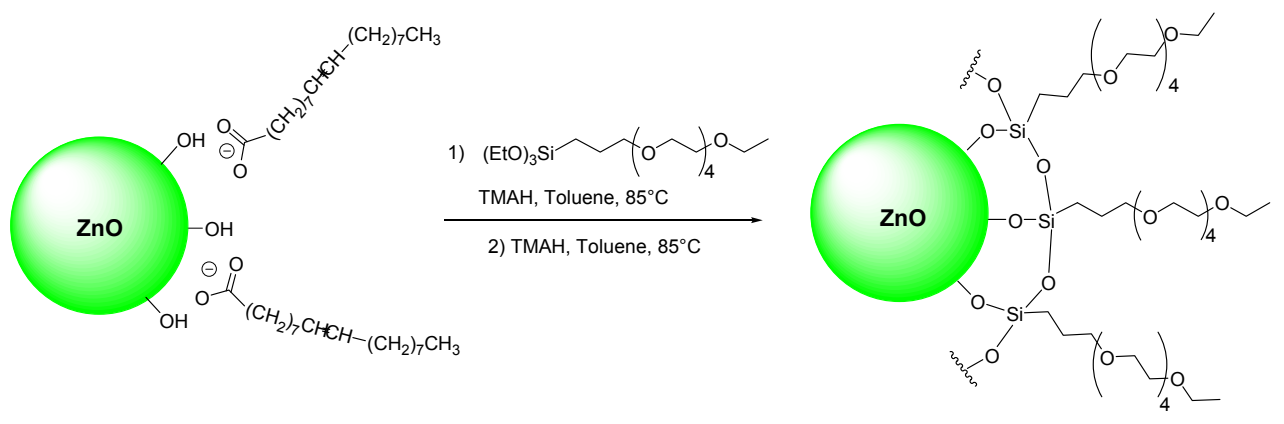

Fig. 10. Synthesis of PEG-siloxane capped $\mathrm{ZnO}$ quantum dots.

Well-dispersed green-emitting ZnO QDs of ca. $6.0 \mathrm{~nm}$ in diameter were obtained after surface functionalization. Their PL QY was found to be $8.0 \%$ in water. $\mathrm{ZnO}$ nanocrystals with mixed functional groups (PEG and amine) could also be prepared using a mixture of the PEG-silane and 3-aminopropyltriethoxysilane in a 4:1 molar ratio during the silanisation step. The amine groups at the periphery of the dots can be used for the covalent attachment of biomolecules allowing these $\mathrm{ZnO}$ QDs to be used as fluorescent labels for ultrasensitive detection and imaging.

To increase the $\mathrm{ZnO}$ materials' flexibility in bio-related applications, we also developed the synthesis of poly(amidoamine) (PAMAM) dendrons bearing a reactive siloxane group at the focal point (Moussodia et al., 2010). These new dendrons were prepared by divergent synthesis through repetitive Michael addition using methyl acrylate and amidation with ethylene diamine starting from 3-aminopropyltrimethoxysilane. Covalent anchorage of the silane function at the surface of ca. $5 \mathrm{~nm}$ diameter ZnO QDs was achieved by treatment of first and second generation dendrons (respectively noted G1 and G2) by an excess of TMAH 
(Figure 11). Due to the presence of polar amine and amide groups and of the high affinity of PAMAM for aqueous media, ZnO@G1 and ZnO@G2 QDs could easily be dispersed in water or phosphate-buffered saline (PBS). ZnO@G1 and ZnO@G2 QDs typically exhibit the greenyellow fluorescence centered at ca. $550 \mathrm{~nm}$ (their PL QYs measured immediately after redispersion in water were found to be 19 and 17\%, respectively). Interestingly, their PL intensities were found to increase upon storage of the dots in water. The PL QY of ZnO@G1 reached $59 \%$ after 20 days, probably due to a surface-ordening of the siloxane-capping, which results in $\mathrm{ZnO}$ QDs with more efficiently protected luminescent centers from water attack.

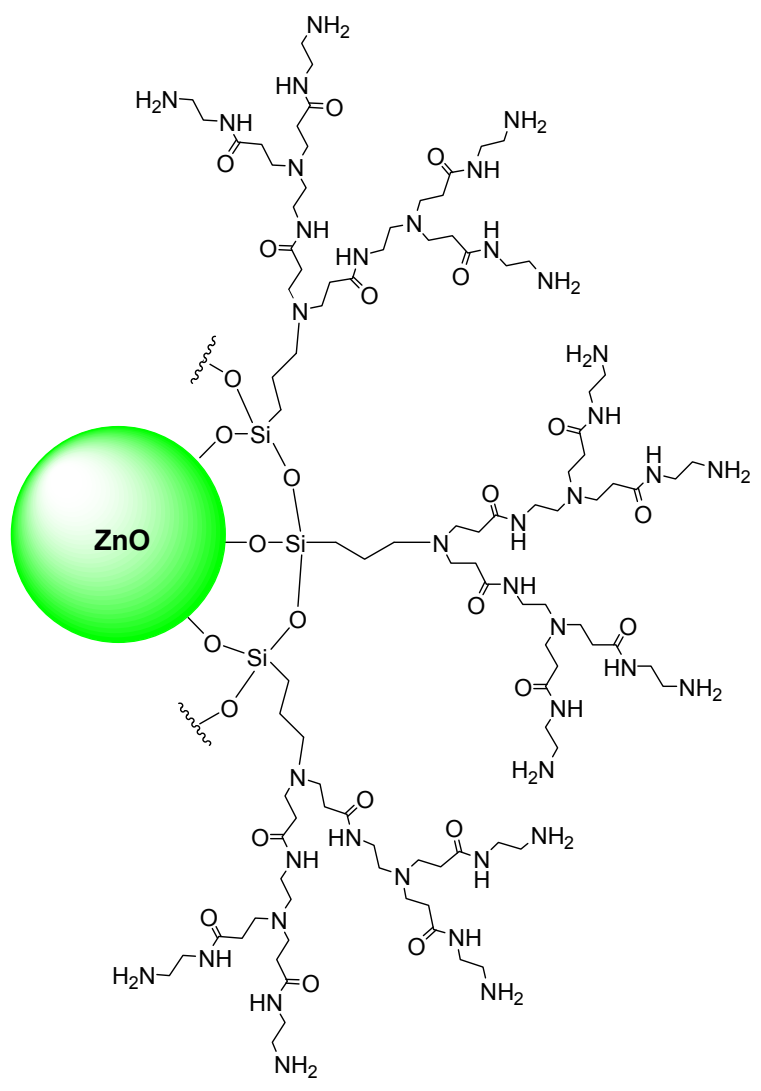

Fig. 11. Structure of $\mathrm{ZnO@G2} \mathrm{quantum} \mathrm{dots.}$

By varying the concentration of the $\mathrm{LiOH}$ base and thus the $\mathrm{pH}$ value of the solution during the sol-gel process, Tang et al. have recently prepared color tunable emission $\mathrm{ZnO}$ QDs. Smaller particles (average diameter of $3.3 \mathrm{~nm}$ ) were formed at $\mathrm{pH}=12$ than at lower $\mathrm{pH}(4.5 \mathrm{~nm}$ at $\mathrm{pH}=10,5.5 \mathrm{~nm}$ at $\mathrm{pH}=8$, and $6.5 \mathrm{~nm}$ at $\mathrm{pH}=6)$ because $\mathrm{ZnO}$ nuclei were formed faster at higher $\mathrm{pH}$ resulting in competition growth. Blue $\left(\lambda_{\mathrm{em}}=455 \mathrm{~nm}\right)$, green $\left(\lambda_{\mathrm{em}}=494 \mathrm{~nm}\right)$, yellow $\left(\lambda_{\mathrm{em}}=570 \mathrm{~nm}\right)$, and orange $\left(\lambda_{\mathrm{em}}=590 \mathrm{~nm}\right)$-emitting ZnO QDs were formed when precipitation values were 12, 10, 8, and 6, respectively (Tang et al., 2010). All 
these nanocrystals exhibit good quantum yields in ethanol (> 20\%) and were stabilized in water by encapsulation into silica nanospheres of ca. $50 \mathrm{~nm}$ in diameter (ca. $20 \mathrm{ZnO}$ QDs per nanosphere). The surface of these nanospheres was further modified with $\mathrm{N}-(2-$ aminoethyl)aminopropyltrimethoxysilane to get positively-charged nanoparticles stable in water from $\mathrm{pH}=4.5$ to 9 . These nanoparticles were successfully used to label $\mathrm{NIH} / 3 \mathrm{~T} 3$ cells and were found to be attached at the cell surface through electrostatic interactions.

The sol-gel method has also successfully been used for the synthesis of $\mathrm{Gd}^{3+}$-doped $\mathrm{ZnO}$ QDs, dual nanoprobes integrating fluorescence and magnetic resonance imaging functionalities into a single nanomaterial (Liu et al., 2011). $\mathrm{Gd}(\mathrm{OAc})_{3}$ and $\mathrm{Zn}(\mathrm{OAc})_{2}$, with $\mathrm{Gd}^{3+} / \mathrm{Zn}^{2+}$ molar ratios varying between 0.02 and 0.3 , were both hydrolysed with TMAH in ethanol in the presence of oleic acid to afford Gd-doped ZnO QDs with diameters ranging from 3.5 to $5.0 \mathrm{~nm}$ depending on the percentage of dopant used (high doping concentration affords the smallest nanocrystals). These Gd-doped ZnO QDs typically exhibit the greenyellow fluorescence related to oxygen vacancies and the highest PL intensity (PL QY $=34 \%$ ) was attained for a $\mathrm{Gd} / \mathrm{Zn}$ molar ratio of 0.08 . These nanocrystals were successfully transferred in aqueous medium through surface functionalization with $\mathrm{N}-(2-$ aminoethyl)aminopropyltrimethoxysilane. MTT cell proliferation assay indicated that Gddoped ZnO QDs were not toxic at concentrations up to $1 \mathrm{mM}$. Gd-doped ZnO QDs were successfully used for the fluorescence imaging of HeLa cells. In MRI studies, they exert strong positive contrast effect with a large longitudinal relaxivity $\left(\mathrm{r}_{1}\right)$ of water proton of 16 $\mathrm{mM}^{-1} \mathrm{~s}^{-1}$.

Luminomagnetic Fe-doped $\mathrm{ZnO}$ nanoparticles with diameters ranging from 6 to $9 \mathrm{~nm}$ were also recently synthesized by treatment of $\mathrm{Zn}\left(\mathrm{NO}_{3}\right)_{2}$ and $\mathrm{Fe}\left(\mathrm{NO}_{3}\right)_{3}$ by potassium hydroxide. The green fluorescent $\mathrm{ZnO}$ nanocrystals doped with ca. $10 \%$ iron exhibit also a paramagnetic behaviour at room temperature (coercivity $=5.1$ milliTesla and magnetization of remanence $=$ 7.6.10-3 emu/g) (Dutta et al., 2010). ZnO:Fe QDs were further dispersed in water through surface functionalization with $\mathrm{N}$-(2-aminoethyl)aminopropyltrimethoxysilane and next conjugated with folic acid using EDC and $N$-hydroxysuccinimide. Because folate receptors are over-expressed on numerous human cells (breast, ovaries, lungs, kidneys, ...), the folic acidconjugated $\mathrm{ZnO}: \mathrm{Fe}$ luminomagnets have great potential for various biomedical applications including bio-labelling, bio-separation, magnetic resonance imaging and targeted drug delivery.

$\mathrm{ZnO}$ QDs with an average diameter of $5 \mathrm{~nm}$ produced by the sol-gel method were also recently used as sensors for the carbohydrate antigen 19-9 (CA 19-9), the preferred label for pancreatic cancer (Gu et al., 2011). A functionalized silicon substrate was first prepared by reaction with 3-aminopropyltrimethoxysilane followed by treatment with glutaraldehyde (Figure 12). An antibody ( $\mathrm{Ab}$ ) monolayer was next assembled on the aldehyde-activated surface and CA 19-9 antigens linked with their antibodies through immunoreaction (samples with various CA $19-9$ concentrations from 0.1 to $180 \mathrm{U} / \mathrm{mL}$ were prepared). In a parallel step, CA 19-9 Abs were immobilized on ZnO QDs through electrostatic interactions in benefit of the high isoelectric point (IEP = 9.5) of $\mathrm{ZnO}$. The sandwich-immunosensor was finally obtained by the $\mathrm{ZnO}-\mathrm{Ab}$ association with the substrate. Detection of CA 19-9 can be performed by fluorescence through measurement of the band-edge emission of ZnO QDs at $372 \mathrm{~nm}$ (linear increase of PL intensity with increase of CA 19-9 concentration), but also through electrochemical measurements, the latter method being more sensitive than photoluminescence. 

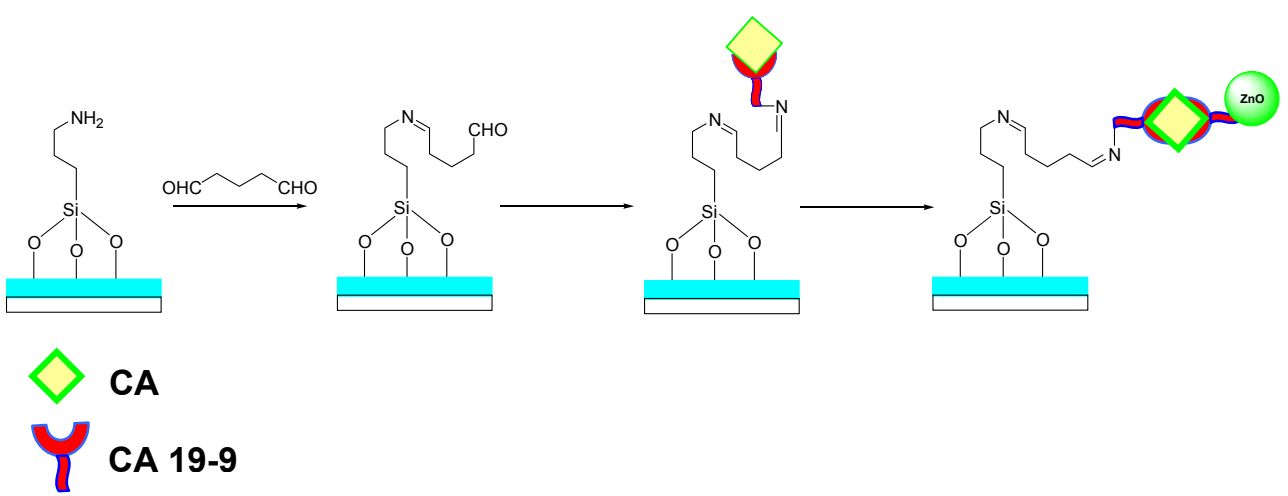

Fig. 12. Schematic protocol of sandwich-immunosensor.

\subsubsection{Cyclodextrin-capped ZnO QDs.}

Another recent example of using ZnO QDs as sensors was reported by Rakshit et al. Carboxymethyl $\beta$-cyclodextrin (CMCD) was used to disperse yellow-emitting core-shell $\mathrm{ZnO} / \mathrm{MgO}$ QDs in aqueous solution (carboxymethyl groups coordinate to the $\mathrm{ZnO}$ surface as carboxylate groups of fatty acids). The surface-anchored cyclodextrins were found to retain their host capabilities for inclusion of small hydrophobic molecules, like the organic dye Nile Red. ZnO/MgO QDs were shown to be excellent donors in fluorescence energy transfer (FRET) to Nile Red. The $640 \mathrm{~nm}$-fluorescence of Nile Red was observed upon excitation of $\mathrm{ZnO} / \mathrm{MgO}$ QDs in the UV (Figure 13). The Nile Red emission following resonance energy transfer exhibits a pronounced thermochromic shift (linear blue shift with increasing temperature) and thus suggest the possible use of the CMCD-capped $\mathrm{ZnO} / \mathrm{MgO}$ QDs-Nile Red assemblies as thermometers in aqueous solution (Rakshit et al., 2008).

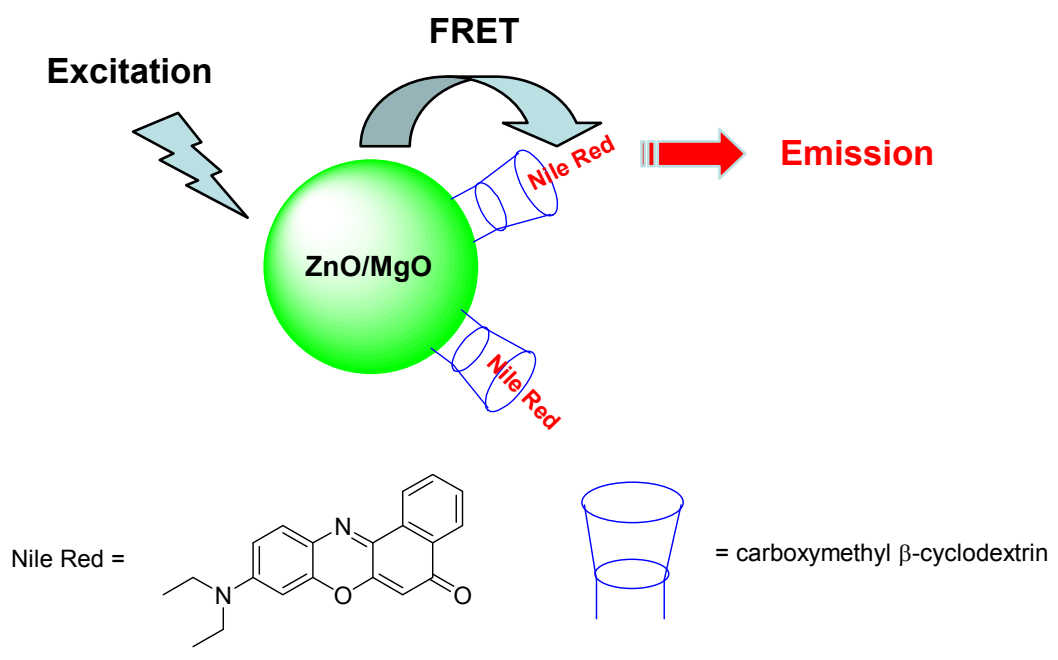

Fig. 13. FRET between the yellow-emitting $\mathrm{ZnO} / \mathrm{MgO}$ QDs and Nile Red included in the cavities of carboxymethyl $\beta$-cyclodextrins. 


\subsubsection{Encapsulation of ZnO QDs into phospholipid micelles.}

$\mathrm{ZnO}$ nanocrystals with a noncentrosymmetric structure produced via non-hydrolytic sol-gel route (Joo et al., 2005) based on the ester-elimination between zinc acetate and 1,12dodecanediol can be used for high contrast nonresonant nonlinear bio-imaging applications (Kachynski et al., 2008). These nanoparticles were dispersed in water using folic acidconjugated phospholipid micelles as stabilizer. Sum frequency generation imaging of human KB cells over-expressing the folate receptor showed a strong internalisation of these $\mathrm{ZnO}$ nanocrystals which have great potential not only as non linear optical probes for diagnostic, but also for the generation of photochemical reactions inside the cells.

\subsection{Decomposition of dialkylzinc for the synthesis of ZnO QDs}

Decomposition/oxidation of organozinc precursors like $\mathrm{Et}_{2} \mathrm{Zn}$ or $\mathrm{Cy}_{2} \mathrm{Zn}$ in the air in the presence of alkylamines or amphiphilic hyperbranched polymers like stearate-modified polyether polyols allows the preparation of $\mathrm{ZnO}$ QDs with control over nanoparticles diameters and photophysical properties (Glaria et al., 2008; Richter et al., 2009). The hydrophobic nanomaterials prepared can however only be dispersed in organic solvents (THF, toluene) and are therefore not suitable for biological applications.

Oxidation of $\mathrm{Cy}_{2} \mathrm{Zn}$ in the presence of hyperbranched polymers (HYPAM) can however give access to water-dispersible ZnO QDs (Saliba et al., 2010). HYPAM were prepared by reaction of a hexaester with a tris-amine and surface functionalized with amphiphilic building blocks formed by alkyldiacids (up to $\mathrm{C}_{18}$ ) connected to monomethyl poly(ethyleneglycol) (mPEG750) (Figure 14).

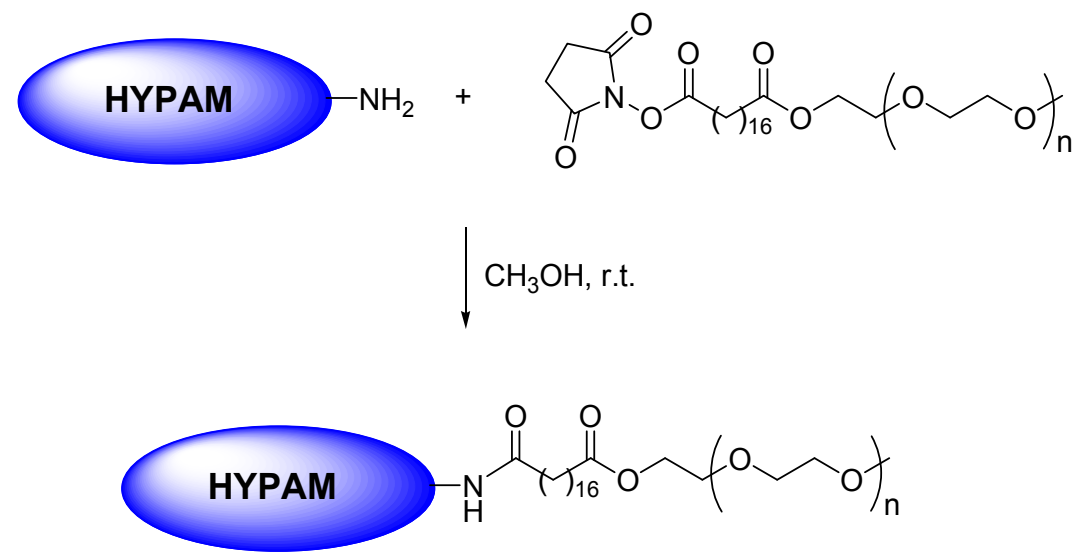

Fig. 14. Preparation of HYPAM polymer with a double-shell structure.

The HYPAM4- $\mathrm{C}_{18}-\mathrm{PEG}_{750}$ polymer has hydrodynamic diameters of ca. 9 and $13 \mathrm{~nm}$ in dilute aqueous solution and in THF solution, respectively. The multiple amine groups of the HYPAM core favour interactions with $\mathrm{Cy}_{2} \mathrm{Zn}$, the inner hydrophobic shell acts as a protective layer for the ZnO QDs produced by hydrolysis, while the outer PEG shell assures their dispersibility in aqueous solution. Depending on the polymer/ $\mathrm{Cy}_{2} \mathrm{Zn}$ weight ratio used for the synthesis, ZnO QDs with diameters ranging from 3.7 to $4.5 \mathrm{~nm}$ can be produced (the hydrodynamic diameter is ca. $30 \mathrm{~nm}$ ). These dots exhibit a broad fluorescence emission centered at $575 \mathrm{~nm}$ but their PL QY is modest (5\%). 


\subsection{Electrolysis for the production of ZnO QDs}

$\mathrm{ZnO}$ nanoparticles of ca. $5 \mathrm{~nm}$ diameter can be prepared by electrochemical deposition under oxidizing conditions using zinc metal as anode. These QDs were first surfacefunctionalized with 3-mercaptopropyltrimethoxysilane (MPTS) and then treated with iodoacetyl-PEG2-biotin. A similar strategy was used for the functionalization of ZnO films (Selegard et al., 2010). Due to the high affinity of the biotin ligand with avidin, these materials have great potential for specific targeting in bio-imaging or for recognition studies in biosensing applications (Figure 15).
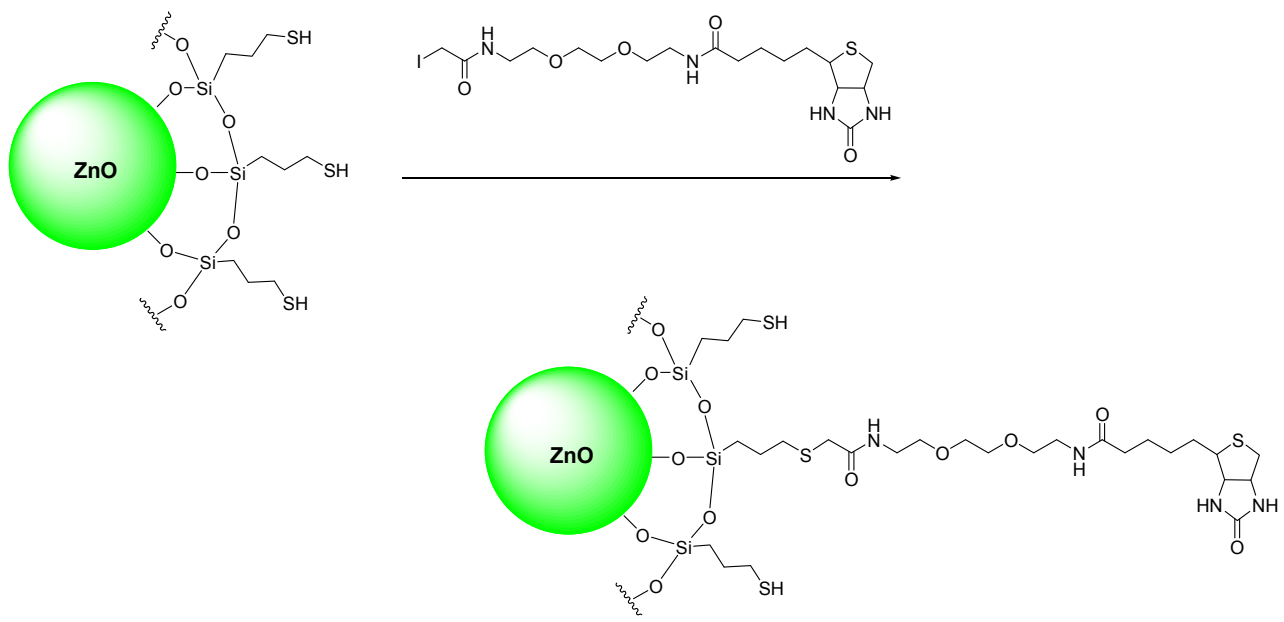

Fig. 15. Surface-functionalization of ZnO@MPTS quantum dots with iodoacetyl-PEG2biotin.

\section{Conclusions}

In recent years, the luminescent properties of $\mathrm{ZnO}$ nanoparticles have attracted considerable attention for numerous applications like ultraviolet light emitting devices, flat panel displays as low voltage phosphor, photovoltaic solar cells, and biosensing devices.

With the developments in fabrication techniques of nanomater-sized, water-dispersible and stable nanocrystals, many efforts have been made to stabilize $\mathrm{ZnO}$ QDs in aqueous medium for biological applications, including employing organic ligands, coating nanoparticles with inorganic shells, and capping nanoparticles surfaces with polymers. In addition, the low toxicity of $\mathrm{ZnO}$ and its high natural abundance make it a good alternative to cadmiumbased II-VI semiconductors, which cause toxicity via photoinduced reactive oxygen species (ROS) generation.

This review highlights recent advances in ZnO QDs synthesis and surface capping for bioapplications and shows that simple and low cost methods have been developed for the production of water stable $\mathrm{ZnO}$ QDs with tunable photoluminescence and high quantum yields. These nanocrystals have successfully been used as fluorescent probes for numerous bio-imaging applications, gene delivery, sensing, and as carrier for targeted drug delivery. All these studies prove that ZnO QDs, as a type of safe and cheap luminescent labels, have an inspiring prospect in biological and medical fields. 


\section{References}

Ahmad, M.; Zhu, J. (2011). ZnO based advanced functional nanostructures: synthesis, properties and applications. J. Mater. Chem., 21, 599-614.

Aldeek, F.; Balan, L.; Lambert, J.; Schneider, R. (2008). The influence of capping thioalkyl acid on the growth and photoluminescence efficiency of CdTe and CdSe quantum dots. Nanotechnology, 19, 475401.

An, S.J.; Park, W.I.; Yi, G.-C.; Kim, Y.-J.; Kang, H.-B.; Kim, M. (2004). Heteroepitaxal fabrication and structural characterizations of ultrafine $\mathrm{GaN} / \mathrm{ZnO}$ coaxial nanorod heterostructures. Appl. Phys. Lett., 84, 3612.

Bahnemann, D.W.; Kromann, C.; Hoffmann, M.R. (1987). Preparation and characterization of quantum size zinc oxide: a detailed spectroscopic study. J. Phys. Chem., 91, 37893798.

Bruce, I.J.; Sen, T. (2005). Surface modification of magnetic nanoparticles with alkoxysilanes and their application in magnetic bioseparations. Langmuir, 21, 7029-7035.

Bruchez, Jr. M.; Moronne, M.; Gin, P.; Weiss, S.; Alivisatos, A.P. (1998). Semiconductor nanocrystals as fluorescent biological labels. Science, 281, 2013-2016.

Chan, W.C.W.; Nie, S. (1998). Quantum dot bioconjugates for ultrasensitive nonisotopic detection. Science, 281, 2016-2018.

Choi, J.H.; Tabata, H.; Kawai, T. (2001). Initial preferred growth in zinc oxide thin films on Si and amorphous substrates by a pulsed laser deposition. J. Cryst. Growth, 226, 493500 .

Costa-Fernandez, J.M.; Pereiro, R.; Sanz-Medel, A. (2006). The use of luminescent quantum dots for optical sensing. Trends Anal. Chem., 25, 207-218.

Ding, Y.; Gao, P.X.; Wang, Z.L. (2004). Catalyst-nanostructure interfacial lattice mismatch in determining the shape of VLS grown nanowires and nanobelts: a case of $\mathrm{Sn} / \mathrm{ZnO}$. J. Am. Chem. Soc., 126, 2066-2072.

Djurisic, A.B.; Leung, Y.H. (2006). Optical properties of ZnO nanostructures. Small, 2, 944961.

Dumas, E.; Gao, C.; Suffern, D.; Bradforth, S.E.; Dimitrijevic, N.M.; Nadeau, J.L. (2010). Interfacial charge transfer between CdTe quantum dots and Gram negative vs Gram positive bacteria. Environ. Sci. Technol., 44, 1464-1470.

Dutta, R.K.; Sharma, P.K.; Pandey, A.C. (2010). Design and surface modification of potential luminomagnetic nanocarriers for biomedical applications. J. Nanopart. Res., 12, 1211-1219.

Glaria, A.; Kahn, M.L.; Cardinal, T.; Senocq, F.; Jubera, V.; Chaudret, B. (2008). Lithium ion as growth-controlling agent of $\mathrm{ZnO}$ nanoparticles prepared by organometallic synthesis. New J. Chem., 32, 662-669.

Gu, B.; Xu, C.; Yang, C.; Liu, S.; Wang, M. (2011). ZnO quantum dot labeled immunosensor for carbohydrate antigen 19-9. Biosens. Bioelectron., 26, 2720-2723.

Jana, N.R.; Yu, H.-h.; Ali, E.M.; Zheng, Y.; Ying, J.Y. (2007). Controlled photostability of luminescent nanocrystalline $\mathrm{ZnO}$ solution for selective detection of aldehydes. Chem. Commun., 1406-1408.

Joo, J.; Kwon, S.G.; Yu, J.H.; Hyeon, T. (2005). Synthesis of $\mathrm{ZnO}$ nanocrystals with cone, hexagonal cone, and rod shapes via non-hydrolytic ester elimination sol-gel reactions. Adv. Mater., 17, 1873-1877. 
Joshi, P.; Ansari, Z.A.; Singh, S.P.; Shankar, V. (2009). Synthesis and characterization of highly fluorescent water dispersible ZnO quantum dots. Adv. Sci. Lett., 2, 360-363.

Kachynski, A.V.; Kuzmin, A.N.; Nyk, M.; Roy, I.; Prasad, P.N. (2008). Zinc oxide nanocrystals for nonresonant nonlinear optical microscopy in biology and medicine. J. Phys. Chem. C, 112, 10721-10724.

Law, W.-C.; Yong, K.-T.; Roy, I.; Ding, H.; Hu, R.; Zhao, W.; Prasad, P.N. (2009). Aqueousphase synthesis of highly luminescent $\mathrm{CdTe} / \mathrm{ZnTe}$ core/shell quantum dots optimized for targeted bioimaging. Small, 5, 1302-1310.

Lim, J.; Jun, S.; Jane, E.; Baik, H.; Kim, H.; Cho, J. (2007). Preparation of highly luminescent nanocrystals and their application to light-emitting diodes. Adv. Mater., 19, 19271932.

Liu, Y.; Ai, K.; Yuan, Q.; Lu, L. (2011). Fluorescence-enhanced gadolinium-doped zinc oxide quantum dots for magnetic resonance and fluorescence imaging. Biomaterials, 32, 1185-1192.

Moussodia, R.-O.; Balan, L.; Schneider, R. (2008). Synthesis and characterization of watersoluble $\mathrm{ZnO}$ quantum dots prepared through PEG-siloxane coating. New J. Chem., 32, 1388-1393.

Moussodia, R.-O.; Balan, L.; Merlin, C.; Mustin, C.; Schneider, R. (2010). Biocompatible and stable $\mathrm{ZnO}$ quantum dots generated by functionalization with siloxane-core PAMAM dendrons. J. Mater. Chem., 20, 1147-1155.

Medintz, I.L.; Uyeda H.T.; Goldman E.R.; Mattoussi, H. (2005). Quantum dot bioconjugates for imaging, labelling and sensing. Nat. Mater., 4, 435-446.

Rakshit, S.; Vasudevan, S. (2008). Resonance energy transfer from $\beta$-cyclodextrin-capped $\mathrm{ZnO}: \mathrm{MgO}$ nanocrystals to included Nile Red guest molecules in aqueous media. ASC Nano, 2, 1473-1479.

Richter, T.V.; Schüler, F.; Thomann, R.; Mülhaupt, R.; Ludwigs, S. (2009). Nanocomposites of size-tunable ZnO-nanoparticles and amphiphilic hyperbranched polymers. Macromol. Rapid Commun., 30, 579-583.

Robel, I.; Subtamanian, V.; Kuno, M.; Kamat, P.V. Quantum dot solar cells. Harvesting light energy with CdSe nanocrystals molecularly linked to mesoscopic $\mathrm{TiO}_{2}$ films. J. Am. Chem. Soc. 2006, 128, 2385-2393.

Saliba, S.; Serrano, C.V.; Keilitz, J.; Kahn, M.L.; Mingotaud, C.; Haag, R.; Marty, J.-D. (2010). Hyperbranched polymers for the formation and stabilization of $\mathrm{ZnO}$ nanoparticles. Chem. Mater., 22, 6301-6309.

Schneider, R.; Wolpert, C.; Guilloteau, H.; Balan, L.; Lambert, J.; Merlin, C. (2009). The exposure of bacteria to CdTe-core quantum dots: The importance of surface chemistry on cytotoxicity. Nanotechnology, 20, 225101.

Selagard, L.; Khranovskyy, V.; Söderling, F.; Vahlberg, C.; Ahren, M.; Käll, P.-O.; Yakimova, R.; Uvdal, K. (2010). Biotinylation of ZnO nanoparticles and thin films: a two-step surface functionalization study. ACS Appl. Mater. Interfaces, 2, 2128-2135.

Spanhel, L.; Anderson, M.A. (1991). Semiconductor clusters in the sol-gel process: quantized aggregation, gelation, and crystal growth in concentrated zinc oxide colloids. J. Am. Chem. Soc., 113, 2826-2833.

van Dijken, A.; Makkinje, J.; Meijerink, A. (2001). The influence of particle size on the luminescence quantum efficiency of nanocrystalline $\mathrm{ZnO}$ particles. J. Lumin. 92, 323-328. 
Soares, J.W.; Whitten, J.E.; Oblas, D.W.; Steeves, D.M. (2008). Novel photoluminescence properties of surface-modified nanocrystalline zinc oxide: toward a reactive scaffold. Langmuir, 24, 371-374.

Tang, X.; Guang Choo, E.S.; Li, L.; Ding, J.; Xue, J. (2010). Synthesis of ZnO nanoparticles with tunable emission colors and their cell labeling applications. Chem. Mater., 22, 3383-3388.

Wu, Y.L.; Lim, C.S.; Fu, S.; Tok, A.I.K. (2007). Surface modifications of ZnO quantum dots for bio-imaging. Nanotechnology, 18, 215604.

Xiong, H.-M.; Wang, Z.-D.; Liu, D.-P.; Chen, J.-S.; Wang, Y.-G.; Xia, Y.-Y. (2005). Bonding polyether onto $\mathrm{ZnO}$ nanoparticles: an effective method for preparing polymer nanocomposites with tunable luminescence and stable conductivity. Adv. Funct. Mater., 15, 1751-1756.

Xiong, H.-M.; Liu, D.-P.; Xia, Y.-Y.; Chen, J.-S. (2005). Polyether-grafted ZnO nanoparticles with tunable and stable photoluminescence at room temperature. Chem. Mater., 17, 3062-3064.

Xiong, H.-M.; Wang, Z.-D.; Xia, Y.Y. (2006). Polymerisation initiated by inherent free radicals on nanoparticle surfaces: a simple method of obtaining ultrastable $(\mathrm{ZnO})$ polymer core-shell nanoparticles with strong blue fluorescence. Adv. Mater., 18, 748-751.

Xiong, H.-M.; Xie, D.-P.; Guan, X.-Y. ; Tan, Y.-J.; Xia, Y.-Y. (2007). Water-stable blue-emitting ZnO@polymer core-shell microspheres. J. Mater. Chem., 17, 2490-2496.

Xiong, H.-M.; Xu, Y.; Ren, Q.-G.; Xia, Y.-Y. (2008). Stable aqueous ZnO@polymer core-shell nanoparticles with tunable photoluminescence and their application in cell imaging. J. Am. Chem. Soc., 130, 7522-7523.

Xiong, H.-M. (2010). Photoluminescent ZnO nanoparticles modified by polymers. J. Mater. Chem. 20, 4251-4262.

Yan, E.; Wang, C.; Wang, S.; Sun, L.; Wang, Y.; Fan, L.; Zhang, D. (2011). Synthesis and characterization of fluorescent chitosan-ZnO hybrid nanospheres. Mater. Sci. Engineer. B, 176, 458-461.

Yuan, Q.; Hein, S.; Misra, R.D.K. (2010). New generation of chitosan-encapsulated ZnO quantum dots loaded with drug: synthesis, characterization and in vitro drug delivery response. Acta Biomater., 6, 2732-2739.

Zhang, L.; Yin, L.; Wang, C.; Lun, N.; Qi, Y.; Xiang, D. (2010). Origin of visible photoluminescence of $\mathrm{ZnO}$ quantum dots: defect-dependent and size-dependent. J. Phys. Chem. C, 114, 9651-9658.

Zhang, P.; Liu, W. (2010). ZnO QD@PMMA-co-PDMAEMA nonviral vector for plasmid DNA delivery and bioimaging. Biomaterials, 31, 3087-3094. 


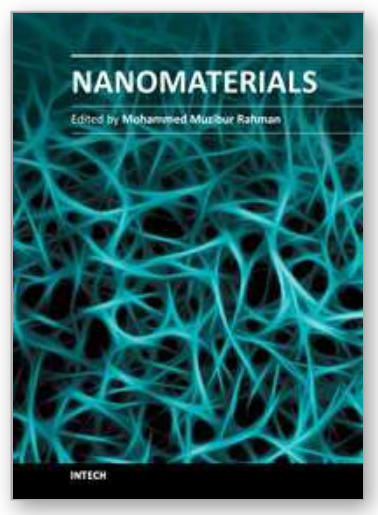

\author{
Nanomaterials \\ Edited by Prof. Mohammed Rahman
}

ISBN 978-953-307-913-4

Hard cover, 346 pages

Publisher InTech

Published online 22, December, 2011

Published in print edition December, 2011

The book "Nanomaterials" includes all aspects of metal-oxide nano-structures, nano-composites, and polymer materials instigating with materials survey and preparations, growth and characterizations, processing and fabrications, developments and potential applications. These topics have utilized innovative methods of preparation, improvement, and continuous changes in multidimensional ways. The innovative frontiers are branching out from time to time to advanced nanotechnology. It is an important booklet for scientific organizations, governmental research-centers, academic libraries, and the overall research and development of nano-materials in general. It has been created for widespread audience with diverse backgrounds and education.

\title{
How to reference
}

In order to correctly reference this scholarly work, feel free to copy and paste the following:

Raphaël Schneider, Lavinia Balan and Fadi Aldeek (2011). Synthesis, Characterization and Biological Applications of Water-Soluble ZnO Quantum Dots, Nanomaterials, Prof. Mohammed Rahman (Ed.), ISBN: 978-953-307-913-4, InTech, Available from: http://www.intechopen.com/books/nanomaterials/synthesischaracterization-and-biological-applications-of-water-soluble-zno-quantum-dots

\section{INTECH}

open science | open minds

\section{InTech Europe}

University Campus STeP Ri Slavka Krautzeka 83/A 51000 Rijeka, Croatia Phone: +385 (51) 770447 Fax: +385 (51) 686166 www.intechopen.com

\section{InTech China}

Unit 405, Office Block, Hotel Equatorial Shanghai No.65, Yan An Road (West), Shanghai, 200040, China 中国上海市延安西路65号上海国际贵都大饭店办公楼405单元 Phone: +86-21-62489820

Fax: +86-21-62489821 
(C) 2011 The Author(s). Licensee IntechOpen. This is an open access article distributed under the terms of the Creative Commons Attribution 3.0 License, which permits unrestricted use, distribution, and reproduction in any medium, provided the original work is properly cited. 